\title{
Severe SMA mice show organ impairment that cannot be rescued by therapy with the HDACi JNJ-26481585
}

\author{
Julia Schreml ${ }^{1,2,3}$, Markus Riessland ${ }^{1,2,3}$, Mario Paterno ${ }^{1,2,3}$, Lutz Garbes ${ }^{1,2,3}$, Kristina Roßbach ${ }^{1,2,3}$, \\ Bastian Ackermann ${ }^{1,2,3}$, Jan Krämer ${ }^{4}$, Eilidh Somers ${ }^{5}$, Simon H Parson ${ }^{5}$, Raoul Heller ${ }^{1}$, Albrecht Berkessel ${ }^{4}$, \\ Anja Sterner-Kock ${ }^{6}$ and Brunhilde Wirth ${ }^{\star, 1,2,3}$
}

\begin{abstract}
Spinal muscular atrophy (SMA) is the leading genetic cause of early childhood death worldwide and no therapy is available today. Many drugs, especially histone deacetylase inhibitors (HDACi), increase SMN levels. As all HDACi tested so far only mildly ameliorate the SMA phenotype or are unsuitable for use in humans, there is still need to identify more potent drugs. Here, we assessed the therapeutic power of the pan-HDACi JNJ-26481585 for SMA, which is currently used in various clinical cancer trials. When administered for $64 \mathrm{~h}$ at $100 \mathrm{nM}$, JNJ-26481585 upregulated SMN levels in SMA fibroblast cell lines, including those from non-responders to valproic acid. Oral treatment of Taiwanese SMA mice and control littermates starting at PO showed no overt extension of lifespan, despite mild improvements in motor abilities and weight progression. Many treated and untreated animals showed a very rapid decline or unexpected sudden death. We performed exploratory autopsy and histological assessment at different disease stages and found consistent abnormalities in the intestine, heart and lung and skeletal muscle vasculature of SMA animals, which were not prevented by JNJ-26481585 treatment. Interestingly, some of these features may be only indirectly caused by $\alpha$-motoneuron function loss but may be major life-limiting factors in the course of disease. A better understanding of - primary or secondary - non-neuromuscular organ involvement in SMA patients may improve standard of care and may lead to reassessment of how to investigate SMA patients clinically. European Journal of Human Genetics (2013) 21, 643-652; doi:10.1038/ejhg.2012.222; published online 17 October 2012
\end{abstract}

Keywords: spinal muscular atrophy; neuromuscular disease; therapy HDAC inhibitors; SMN2; JNJ-26481585; VPA

\section{INTRODUCTION}

Proximal spinal muscular atrophy (SMA) is a neuromuscular disorder characterized by progressive atrophy and weakness of proximal voluntary muscles due to the loss of $\alpha$-motoneurons in the spinal cord. ${ }^{1}$ With an incidence of 1 in 6000 live-births and a carrier frequency of 1 in 35 in the Caucasian population, it is the leading genetic cause of infant death. ${ }^{2,3}$ SMA is caused by functional loss of the survival of motor neuron gene 1 (SMN1) due to homozygous deletion, gene conversion or mutations. ${ }^{4-6}$ The severity of SMA has been traditionally classified by the clinical presentation as ranging from severe type I to very mild type IV with respect to age of onset and maximum motor function achieved. ${ }^{7}$ The disease course is mainly influenced by the number of SMN2 copy genes. ${ }^{2,8,9}$ In comparison with SMN1, SMN2 carries a translationally silent mutation that affects the correct pre-mRNA splicing of exon $7 .^{10-12}$ This leads to the production of only $\sim 10 \%$ full-length (FL) SMN2 transcript and protein identical to the protein encoded by $S M N 1 .^{4,13}$ About $90 \%$ of SMN2 transcripts, however, lack exon 7 (SMN2 47 ) and encode a truncated and unstable protein. ${ }^{11,14}$

SMA is quite unique among inherited diseases, not only because of the possibility to activate the SMN2 copy gene but also because of the ease of screening for SMN1 deletions, which are found in $94 \%$ of patients. ${ }^{5}$ A therapy leading to reliable upregulation of SMN2 transcription can therefore be expected to be effective in most SMA patients. Hence, many therapeutic strategies have been suggested and reported, including: (i) elevation of SMN2 RNA and protein levels by transcription activation, correction of SMN2 splicing or stabilization of protein using drugs or antisense oligos, (ii) gene replacement by gene therapy approaches, (iii) neuroprotective therapy and (iv) stem cell therapy (reviewed in ${ }^{15-17,18}$ ). Despite these promising experimental approaches, no readily available effective treatment for SMA exists to date. ${ }^{19,20}$ Targeting SMN2 gene transcription by use of histone deacetylase inhibitors (HDACi) such as valproic acid (VPA), Trichostatin A, SAHA, sodium butyrate, LBH589 or M344 ${ }^{21-28}$ is an approach with potentially fast clinical implementation. Moreover, HDACi treatment has previously been shown to increase SMN2 transcription and to ameliorate the phenotype in SMA mouse models. ${ }^{27,29-31}$ However, the clinical benefit of the substances tested to date remains uncertain..$^{21,32-35}$ A complete rescue of the phenotype by HDACi application is unlikely to be achieved for patients most severely affected and only possessing a low number of SMN2 copies, and thereby restricted potential for increase in FL protein expression. Nonetheless, improvement of quality of life is still an objective worth investigating for these patients.

In the present work, we studied the pan-HDACi JNJ-26481585, which is currently subject of multiple phase I and II antineoplastic

${ }^{1}$ Institute of Human Genetics, University of Cologne, Cologne, Germany; ${ }^{2}$ Institute for Genetics, University of Cologne, Cologne, Germany; ${ }^{3}$ Center for Molecular Medicine Cologne, University of Cologne, Cologne, Germany; ${ }^{4}$ Department of Chemistry, University of Cologne, Cologne, Germany; ${ }^{5}$ Euan MacDonald Centre for Motor Neurone Disease Research and Centre for Integrative Physiology, University of Edinburgh, Edinburgh, Scotland; ${ }^{6}$ Department of Experimental Medicine, University of Cologne, Cologne, Germany *Correspondence: Professor B Wirth, Institute of Human Genetics, University of Cologne, Kerpener Street 34,50931 Cologne, Germany. Tel: +49 221478 86464; Fax: +49 221478 86465; E-mail: brunhilde.wirth@uk-koeln.de

Received 29 March 2012; revised 28 August 2012; accepted 6 September 2012; published online 17 October 2012 
clinical trials (http://www.clinicaltrials.gov/ct2/results?term=JNJ26481585)..$^{36,37}$ JNJ-26481585 is a hydroxamic acid derivative, chemically related to LBH589, which is the most potent HDACi in the upregulation of SMN protein in human patient fibroblast cell lines studied so far. ${ }^{26}$ The improved pharmacological profile and increased half-life of JNJ-26481585 as compared with LBH589 prompted us to test this compound in vitro and in vivo for its benefit on SMA. 38,39

For in vivo analysis, we used a severe SMA mouse model developed by a Taiwanese group. ${ }^{40}$ An efficient breeding scheme $\left(S m n^{-1-}\right.$; $S M N 2^{t g / t g} \mathrm{X} \mathrm{Smn}^{+1-}$ ) developed in our laboratory resulted in $50 \%$ of each litter developing SMA $\left(S m n^{-1-} ; S M N 2^{t g / 0}\right)$ with a mean survival of $\sim 10$ days on pure $\mathrm{FVB} / \mathrm{N}$ background. The remaining $50 \%$ are healthy SMA carriers $\left(S m n^{+/-} ; S M N 2^{\mathrm{t} g / 0}\right)$ and used as controls. ${ }^{30}$

Recent reports suggest that abnormal heart development in mice and human ${ }^{41-44}$ as well as defects in skeletal muscle ${ }^{45}$ and skeletal muscle vasculature ${ }^{46}$ of mice are part of the phenotype and that SMA may be not a pure motoneuron disease. In addition, decreased levels of hepatic insulin-like growth factor binding protein acid labile subunit ${ }^{18}$ possibly account for some of the dwarfism observed consistently across different SMA mouse models. Here, we show that SMA mice exhibit abnormalities of several internal organs, particularly lung, intestine and heart as well as skeletal muscle capillary defects. JNJ-26481585 therapy increased SMN levels in spinal cord, improved motor abilities and weight gain. However, it could not rescue internal organ pathology and reduced skeletal muscle capillarity, thus explaining cases of sudden death and lack of extension of lifespan observed in SMA animals. Our findings extend the spectrum of organs affected in SMA mice. If proven in further studies to be primary effects of diminished SMN levels rather than secondary evolving pathologies, this underpins the need for systemic SMN upregulation and a multimodal approach for longterm therapy.

\section{MATERIALS AND METHODS}

\section{Cell culture and treatment of cell lines}

Primary human SMN1-deleted fibroblasts cell lines were established from skin biopsies derived from SMA patients who fulfilled the diagnostic criteria for SMA and were cultured as described elsewhere. ${ }^{22,26}$ Informed written consent was obtained from all patients according to the Declaration of Helsinki and the study has been approved by the ethical committee of the University Hospital of Cologne. Primary murine embryonic fibroblast cell lines were established from SMA mouse embryos harvested at embryonic day 13.5 as described elsewhere. $^{47}$

For drug treatment the day before treatment either some $2 \times 10^{5}$ fibroblasts were seeded into $10 \mathrm{~cm}$ dishes or some $1 \times 10^{5}$ cells in 6-well plates, respectively. Cells were treated with various concentrations (10 nM-1 $\mu \mathrm{M})$ of JNJ-26481585 (patent number US2010/0130523 A1, synthesized in the Department of Chemistry, University of Cologne, Germany by Jan Krämer and Prof. Albrecht Berkessel) dissolved in DMSO. Following $16-72 \mathrm{~h}$ of treatment, subconfluent fibroblasts were harvested. LBH589 (Novartis, Basel, Switzerland) and VPA (Sigma-Aldrich, Munich, Germany) treatment was performed as described previously. ${ }^{22,26}$

\section{Determination of transcript levels by qRT-PCR}

To isolate total RNA from fibroblast cell culture, the RNeasy Kit (Qiagen, Hilden, Germany) and the QIAshredder (Qiagen) were used according to the manufacturer's protocols. Subsequent analyses of FL-SMN2 and $\triangle 7 S M N 2$ transcripts by quantitative real-time RT-PCR (qRT-PCR) on a LightCycler 1.5 (Roche, Basel, Switzerland) were performed as previously described. ${ }^{25}$

\section{Cell viability assay}

To assess the number of viable cells, an MTT assay was performed as described previously. ${ }^{25}$ In brief, MTT, a tetrazole, is reduced to formazan by mitochondrial reductases in active proliferating cells. Spectrophotometric quantification of formazan amounts allowed the determination of cell viability.

\section{SMN ELISA}

ELISA was performed using an SMN ELISA-kit (Enzo Life Sciences, Lörrach, Germany) as described in the SOP 'SMA M.1.2.005' brought forth by the Treat-NMD Neuromuscular Network (http://www.treat-nmd.eu/resources/ research-resources/sma-sops/). A standard curve was established for each assay using the provided SMN standard solution, and protein concentration of samples was read against the standard curve.

\section{Mice}

We used the Taiwanese SMA mouse model, FVB.Cg-Tg(SMN2)2Hung $S m n 1^{\text {tmlHung } / J}$ purchased from Jackson's Laboratory (Bar Harbor, ME, USA; Stock Number: 005058). SMA mice are described in detail and were bred as previously reported with $50 \%$ of the offspring being SMA mice $\left(S m n^{-1-} ; S M N 2^{\text {tg/0 }}\right)$ and $50 \%$ control carriers $\left(\mathrm{Smn}^{-1+} ; \mathrm{SMN}^{\mathrm{tg} / 0}\right){ }^{30}$ Mice carrying homozygously the SMN2 transgene on exon 7 disrupted murine Smn background $\left(\mathrm{Smn}^{-1-}\right.$; $\left.S M N 2^{\operatorname{tg} / \operatorname{tg}}\right)^{40}$ fail to develop any motor SMA phenotype, are fertile and live for $>1$ year, but develop a short and thickened necrotic tail and necrotic ears. Mice heterozygous for the SMN2 transgene on exon 7 disrupted murine Smn background $\left(S m n^{-1-} ; S M N 2^{t g / 0}\right)$ developed a severe SMA phenotype with a mean age of survival of $\sim 10$ days. As a wild-type strain, the inbred strain FVB/ NJ (Jackson's Laboratory, Stock Number: 001800) was used. The Taiwanese SMA mice were housed in micro-isolation chambers in the mouse facility of the Center of Molecular Medicine Cologne, Cologne. All mice were humanely euthanized according to protocols set forth by the 'Landesamt für Natur, Umwelt und Verbraucherschutz NRW'.

The animal breeding and all mouse experiments were approved by the local animal protection committee. The animal experiment application form was confirmed. All in vivo experiments were performed under the reference number 9.93.2.10.31.07.292. Mice were treated orally using a feeding needle starting with postnatal day zero (P0). Doses for the various experiments are given in the result section.

\section{Genotyping}

For each animal used in this work, the genotype was analyzed by previously described genotyping PCR reactions on respective tail-tip DNA. Primer sequences and PCR conditions were used as previously described. ${ }^{40}$

\section{Evaluation of disease progression}

To assess progression of the disease phenotype, mice were weighted daily in the morning. To characterize motor abilities of the animals, the tube test was applied as described in detail elsewhere. ${ }^{48}$ In summary, each pup was placed headfirst into a vertical $50 \mathrm{ml}$ reaction tube containing some bedding at the bottom of the tube. Motor performance was scored on a scale of $0-4$ regarding the ability of the animals to hold themselves using their hindlimbs in an active manner as indicated by the spreading of the hindlimbs, elevation of the tail and time they were able to hold on to the rim of the tube. A mouse was rated with the worst score of 0 when it was not able to hold on to the tube. The tube test was performed daily starting at P0. General observations regarding the health such as occurrence of diarrhea and special findings of individual animals were noted as appropriate on a daily basis.

\section{Preparation of proteins from mouse organs}

For the preparation of mouse organs, mice were killed by decapitation at P0, P5 or day P8, respectively. Mice were fixed on an operating dish and the skin including subcutaneous fat was removed from the belly leaving the peritoneum and the abdominal muscular layers intact. Mice were examined for macroscopic abnormalities such as abdominal fluid, then the abdominal cavity was opened and organs were removed quickly. For protein preparation of organs, whole spinal cord, brain and liver were removed and organs were immediately 
snap-frozen and stored in liquid nitrogen. Tissue lysates were homogenized by the use of an Ultra Turrax homogenizer (IKA) in RIPA buffer (Sigma-Aldrich).

\section{Western blot analysis}

Western blot analysis was performed as previously described. ${ }^{22}$ Mouse monoclonal anti- $\beta$-actin (1:10 000, Sigma-Aldrich) and mouse monoclonal anti-SMN (1:1000, BD Transduction laboratories, Franklin Lakes, NJ, USA) were used.

\section{Histology}

For subsequent histological analyses, biopsies from the gastrocnemius muscle and the rectus femoris muscle were taken as well as from the heart, lungs and the intestine. All organs for histological stainings were immediately fixed in $4 \%$ paraformaldehyde and stored at $4{ }^{\circ} \mathrm{C}$ over night. Whole mouse organs or biopsies were dehydrated using an infiltration machine (Leica, Wetzlar, Germany) and subsequently embedded in paraffin. Embedded samples were cut using a microtome (Leica) to produce $5 \mu \mathrm{m}$ thick sections. Haematoxylin/eosin staining was performed. Intestinal wall edema was assessed using a scoring system of five categories ranging from massive $(++++)$ through severe $(+++)$, moderate $(++)$ to mild/possible $(+)$ and no visible edema $(-)$. Scoring was performed on a minimum of 15 sections per animal with a minimum of five individual slides. Mean values are given per animal.

\section{Immunostaining of NMJs}

Immunostaining of NMJs was performed on whole mount transversus abdominis (TVA) preparations. For TVA preparations, skin was carefully removed on the front and back, leaving the musculature and peritoneum intact. Suprapubic bilateral incisions were made from the midline of the abdomen reaching to the spine on both sides. Incisions were extended proximally on the back of the mouse, separating the ribcage from the spine. Abdominal organs including the diaphragm were carefully removed, so that the thoracic cage including inferiorly the entire TVA muscle was removed, rinsed with $1 \times$ PBS and fixed on a Petri dish for further preparation under a Leica binocular. TVA musculature was prepared and fixed in PFA $4 \%$ for $20 \mathrm{~min}$, then washed and stored in $1 \times$ PBS until staining. Anti-Neurofilament M (subsequently stained with a donkey anti-mouse antibody labeled with AlexaFlour 488, Millipore, Billerica, MA, USA), which labels the neurons, and $\alpha$-Bungarotoxin (labeled with rhodamine, Invitrogen, Carlsbad, CA, USA), which binds to the muscular acetylcholine receptors, were used. The surface area of at least $100 \mathrm{NMJs}$ per animal was measured with the help of Axio Vision computer software (Zeiss, Oberkochen, Germany).

\section{Immunostaining of capillary bed}

TVA muscle was prepared and fixed as described above. Capillary bed staining was carried out as described in Somers et al. ${ }^{46}$ Following PBS washing, muscles were incubated with Rhodamine-labeled Griffonia Simplicifolia Lectin I (GSL I, BSL 1) (1:100 diluted in $0.1 \mathrm{~m}$ PBS, Vector Laboratories, Burlingame, CA, USA) for $1 \mathrm{~h}$ at room temperature, washed and then mounted in Mowiol on slides. Images were taken using an inverted Olympus I $\times 71$ microscope $(\times 40$ objective, $0.8 \mathrm{NA}$ ) a chilled CCD camera (Hammamatsu, Hammamatsu, Japan; C4742-95) and OpenLab (Improvision) image capture software. For all muscles a minimum of 40 images per muscle were taken. Generation of a value for capillary area in $\mathrm{mm}^{2}$ per $\mathrm{mm}^{2}$ of muscle was performed as previously described. ${ }^{46}$

\section{Statistical analysis}

The significance of the RNA expression levels as well as of the changed protein levels was tested using a directional student's $t$-test for uncorrelated samples. In all cases, three levels of statistical significance were distinguished: $P<0.05\left(^{*}\right)$,
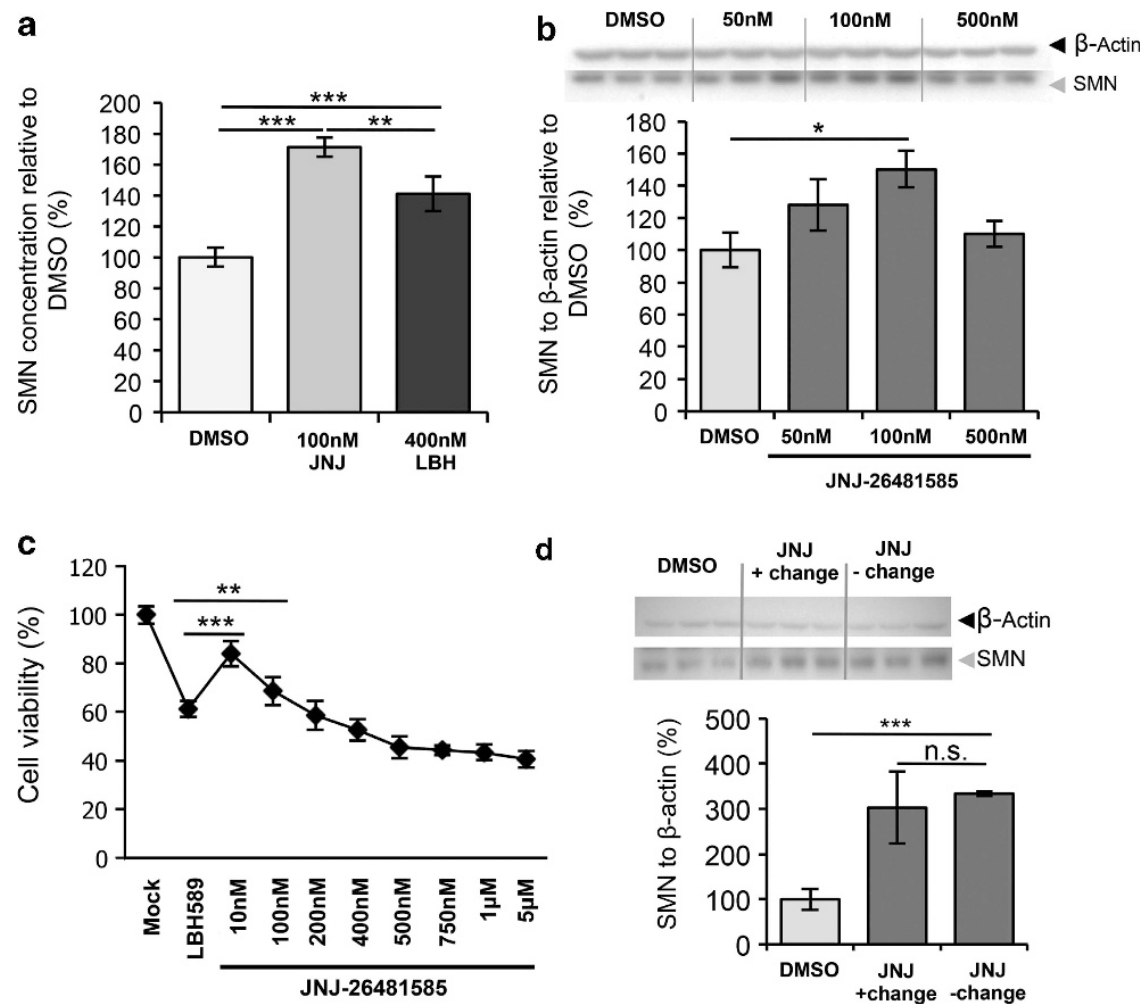

Figure 1 Efficacy and toxicity of JNJ-26481585 in primary patient fibroblasts and primary murine embryonic fibroblasts (MEFs). (a) SMN ELISA - JNJ-26481585 versus LBH589 in human SMA primary fibroblast cell line ML112. JNJ-26481585 surpasses efficiency of LBH589 treatment. Incubation for $64 \mathrm{~h}$. (b) Western

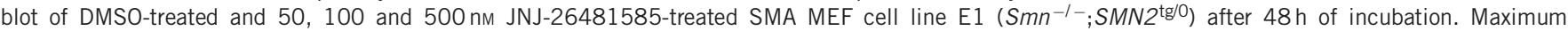
induction was reached at $100 \mathrm{~nm}$. (c) MTT toxicity assay using the human SMA primary fibroblast cell line ML100 and treatment with $400 \mathrm{~nm}$ LBH589 and a range of concentration of JNJ-26481585 between $10 \mathrm{~nm}$ and $5 \mu \mathrm{m}$. (d) Western blot of JNJ-26481585 after $48 \mathrm{~h}$ with $(+)$ and without $(-)$ change of medium to withdraw drug exposure after $24 \mathrm{~h}$ incubation in human SMA primary fibroblast cell line ML17. JNJ-26481585 shows sustained activity in absence of continuous drug exposure. Results for $\mathbf{a}, \mathbf{b}$ and $\mathbf{d}$ in triplicates. Results for $\mathbf{c}$ in octuplicates. Bars show mean $\pm \mathrm{SD},{ }^{*}=P<0.05,{ }^{* *}=P<0.01,{ }^{* * *}=P<0.001$. 
$\left.P<0.01{ }^{* *}\right)$ and $\left.P<0.001{ }^{* * *}\right)$. The significance of the differences in NMJ surface area size was determined by the use of a directional student's $t$-test for uncorrelated samples. All statistical analyses were performed using Excel 2003 (Microsoft, Redmond, WA, USA), Sigma Plot 9.0 (Systat Software, Erkrath, Germany) or ANOVA.

\section{RESULTS}

JNJ-26481585 shows prolonged activity and upregulates SMN protein levels in patient primary fibroblast cell lines at doses with low toxicity

JNJ-26481585, a second-generation HDAC inhibitor was previously described to show prolonged pharmacodynamic response in vivo. ${ }^{38,39}$ Its marked potency $\left(\mathrm{IC}_{50}=0.16 \mathrm{nmol} / \mathrm{l}\right)$ as well as its continued activity in solid tissue makes it an interesting candidate for SMA therapy. ELISA analysis showed that JNJ-26481585 is more effective at a $4 \times$ lower dose of $100 \mathrm{nM}$ for $64 \mathrm{~h}$ in upregulating SMN2 protein expression than LBH589, a structurally similar HDACi, shown to be highly efficient in upregulation of $\mathrm{SMN}^{26}$ (Figure 1a). Treatment of primary human SMA fibroblast cell lines with higher doses did not result in additional upregulation of SMN protein expression at 48-64 h treatment (data not shown). Next, we demonstrated by western blot analysis that in contrast to VPA, which failed to upregulate SMN in about $2 / 3$ of SMA patient cell lines, ${ }^{21}$ JNJ-26481585 is able to overcome VPA nonresponsiveness (Supplementary Figure S1A and 1B). Quantification of $F L-$ and $\triangle 7 S M N 2$ transcripts by RT-PCR revealed upregulation of both isoforms in one case (ML 86) and upregulation of FL-SMN2 and downregulation of $\triangle 7 S M N 2$ in two fibroblast cell lines (Supplementary Figure S1B). Western blot analysis performed on murine embryonic fibroblast cell culture derived from an SMA mouse embryo with doses of 50, 100 and $500 \mathrm{~nm}$ concentrations confirmed maximum effect of SMN2 upregulation in the murine setting at doses of $100 \mathrm{~nm}$ (Figure 1b). At the dose of maximum effect (10-100 nм for 48-64 h in human fibroblasts) JNJ-26481585 showed less toxicity than LBH589 ( $400 \mathrm{~nm}$ ) as shown in the MTT assay (Figure 1c). We further demonstrated that the reported prolonged drug response was also true for SMN protein upregulation, as the effect was retained after $24 \mathrm{~h}$ drug removal compared with continuous exposure over $48 \mathrm{~h}$ (Figure 1d). On the basis of these results as well as previous work on other HDACi, we went straight forward to analyze JNJ-26481585 in vivo, using SMA mice.

JNJ-26481585 mildly ameliorates the motor and weight phenotype of SMA mice but cannot overcome early death in these animals We used the Taiwanese SMA mouse model for in vivo analysis of JNJ26481585. Breeding of $S m n^{-1-} ; S M N 2^{\text {tg/tg }}$ with $S m n^{+/-}$resulted in $50 \%$ SMA $\left(\mathrm{Smn}^{-l-}\right.$; SMN2 $\left.2^{\mathrm{tg} / 0}\right)$ and 50\% control carriers $\left(\mathrm{Smn}^{-l+}\right.$; $\left.S M N 2^{\mathrm{tg} / 0}\right)$ in each litter. ${ }^{30}$ We found that doses of $10 \mathrm{mg} / \mathrm{kg}$ daily and $5 \mathrm{mg} / \mathrm{kg}$ daily of JNJ-26481585 (dissolved in DMSO) were toxic leading to death before P5 and P8, respectively, irrespective of the genotype. Doses as low as $1.25 \mathrm{mg} / \mathrm{kg}$ every 5 th day starting at P0 led to a mild improvement of the phenotype as seen by significantly better motor test results at P8 $(P<0.001)$ (Figure 2b) and significantly higher weight at P8, P9 $(P<0.001)$ and P10 $(P=0.01)$ compared with untreated animals (Figure 2a). In untreated animals, weight gain began to lag behind as early as P3 in some animals and became overt on P5 whereas - with few exceptions - drug-treated animals showed weight progression until at least P10 (Figure 2a) but remained small compared with control littermates. Motor deficits became prominent around P6 or P7 in untreated and around P10 in treated animals (Figure 2b). Augmentation of the dose up to $2.5 \mathrm{mg} / \mathrm{kg}$ starting at P6 and treatment every other day did not result in any quantifiable additional outward improvement of the phenotype. Despite transient improvement in motor skills and weight progression,
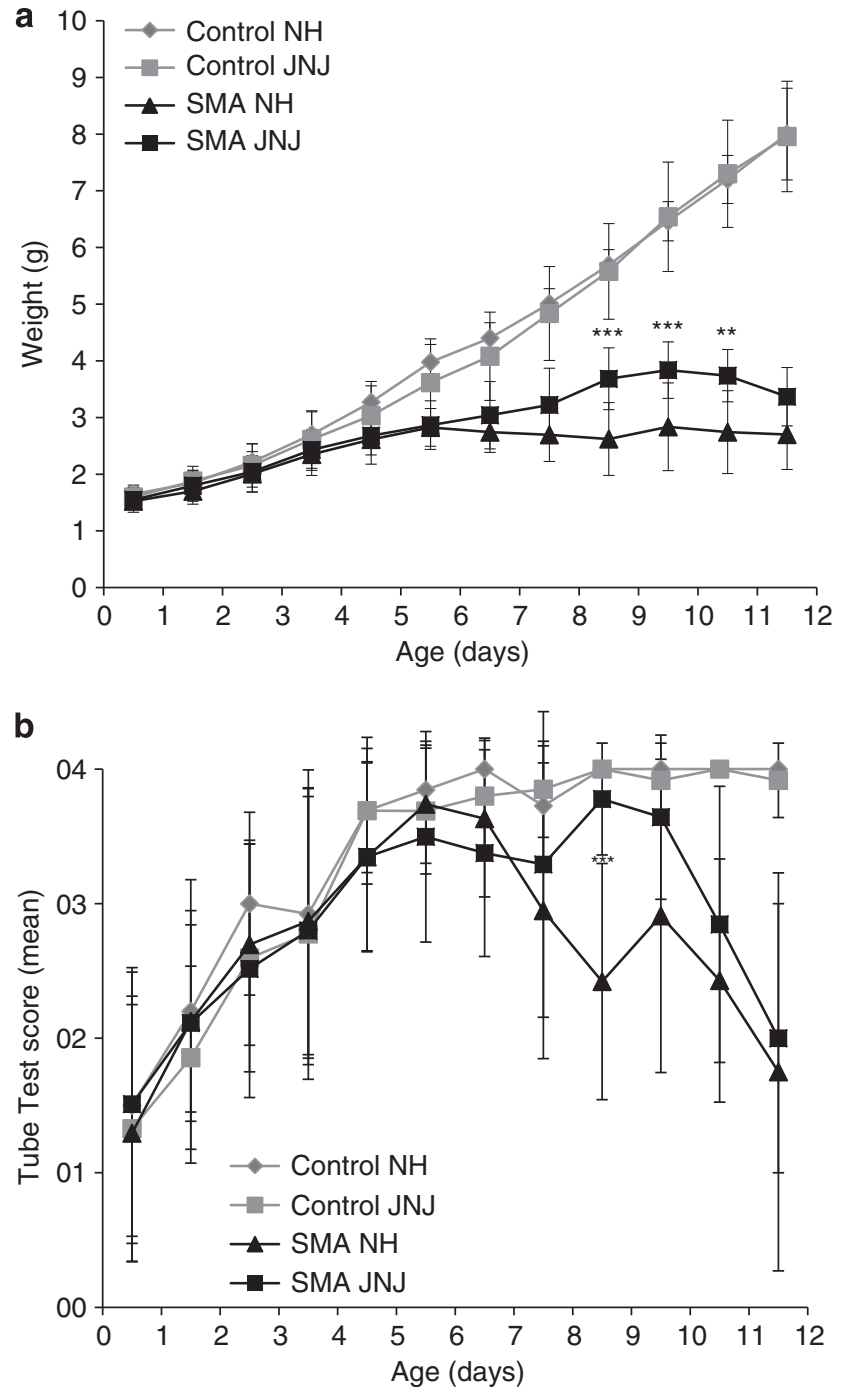

Figure 2 Improvement of motor performance and weight progression in SMA animals under JNJ-26481585 treatment. (a) Untreated ( = Natural History $=\mathrm{NH}$ ) SMA animals stop in their weight progression around P5 whereas treated animals continue to gain weight until P9 with significant differences between $\mathrm{P} 8$ and P10. (b) Untreated SMA NH animals show a rapid decline in tube test performance. JNJ-26481585-treated animals sustain motor performance until P9; $N=19$ (untreated), $N=18$ (JNJ-26481585-treated). Bars show mean $\pm \mathrm{SD},{ }^{* *}=P<0.01,{ }^{* * *}=P<0.001$.

both untreated and JNJ-26481585-treated animals showed a mean survival of $\sim 11$ days across all treatment schemes. Moreover, many animals died before any evident phenotype was observed in the tube test. In the light of our treatment trials not showing sufficient response, we prematurely aborted dose finding and collection of respective survival data, but went on to assess the upregulation of SMN levels in vivo in different key organs and to explore possible causes for the unexplained sudden deaths observed in SMA animals.

JNJ-26481585 shows upregulation of SMN protein levels in vivo To investigate whether SMN levels increase under JNJ-26481585 treatment $(2.5 \mu \mathrm{g}$ at $\mathrm{P} 0$ and $\mathrm{P} 2$ and dose escalation to $5 \mu \mathrm{g}$ every other day from P4), we performed western blot analysis from spinal cord, brain and muscle using JNJ-26481585 or untreated SMA 
animals and controls at P5 and P8. Significant upregulation in SMA animals (brain $P=0.01$, spinal cord $P=0.018$ ) was observed at $\mathrm{P} 8$ but not P5 in spinal cord and brain (Figures $3 \mathrm{Ac}$ and $\mathrm{Bc}$ ). No upregulation was seen in quadriceps femoris (QF) muscle (Supplementary Figure S2). These data indicate that JNJ-26481585 is capable of passing the blood-brain barrier and upregulating SMN levels in the spinal cord - the target tissue - and brain, but not in the QF muscle.

SMA mice exhibit marked abnormalities in multiple organs even before motor deficits become apparent

Several JNJ-26481585-treated animals, and some untreated animals, died unexpectedly while apparently otherwise in relatively good health and showing little or no motor deficits. We observed diarrhea in SMA animals as early as P6 and virtually all SMA animals were affected by P8. Diarrhea was less pronounced in treated animals (Table 1).
Exploratory autopsy on drug-treated and untreated mice at different ages revealed - irrespective of treatment - at P8 spotted dark red discolorations of the lungs compatible with atelectasis or pulmonary infarctions (Supplementary Figure S3F and G). Moreover, the intestine appeared empty and string-like and opaque fluid was found in the abdomen of most late-stage SMA animals (Supplementary Figure S3C) but not in control littermates (Supplementary Figure S3D). Cardiac histology suggested interventricular septum (IVS) thinning (Figure 4B) in accordance with previous observations for the $\Delta 7$ mouse model and the severe SMA mouse model containing two SMN2 copies. ${ }^{43}$ Respective differences were present at $\mathrm{P} 5$, a time point before any motor phenotype was observed (Figure 2b). Pulmonary lesions in SMA animals were characterized by variable degrees of emphysema with ruptured alveolar septa and enlarged alveolar spaces compared with heterozygous animals (Figures 4Ac and g). Histological changes in the small intestine
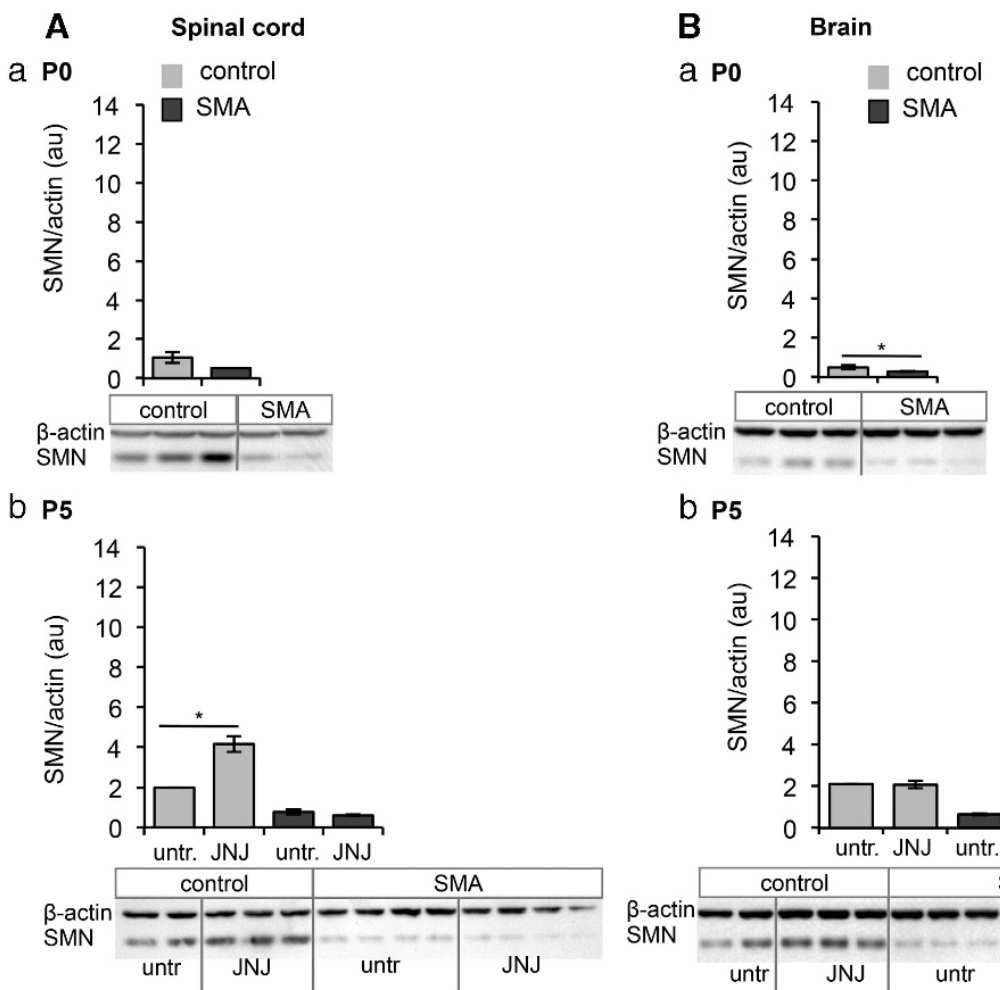

b P5

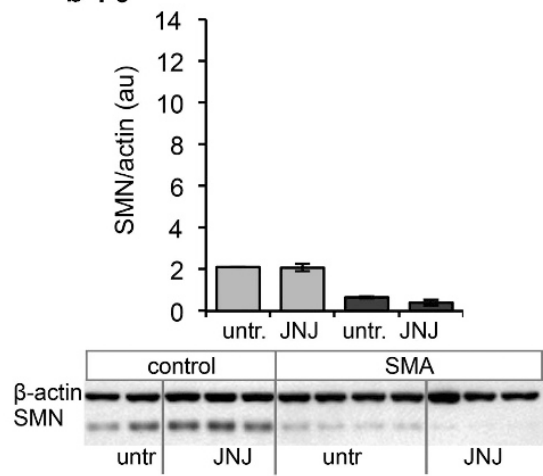

C P8

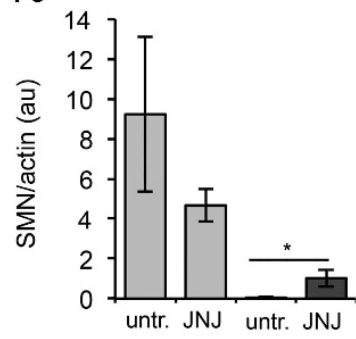

C P8
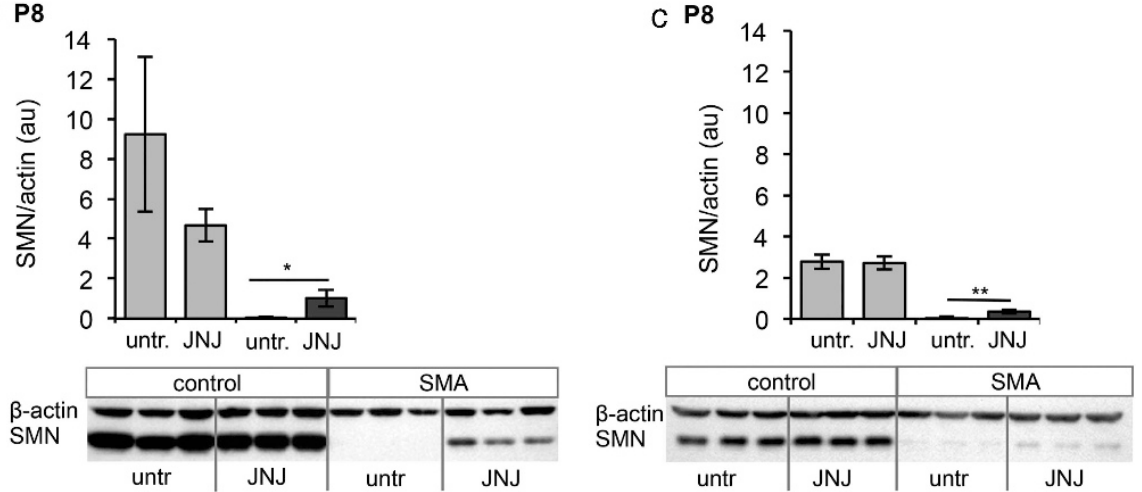

Figure 3 SMN protein level in the spinal cord and brain of JNJ-26481585-treated vs untreated SMA and control littermates at different disease stages. Western blot analysis of the change of SMN levels normalized to $\beta$-actin in the spinal cord (A) and brain (B) of SMA and control littermates at P0, P5 and P8 (a-c). Note: the $\alpha$-SMN antibodies cannot discriminate between mouse Smn and human SMN in controls. Untreated animals were compared with animals treated with a dose of $2.5 \mu \mathrm{g}$ at $\mathrm{P} 0$ and $\mathrm{P} 2$ and $5 \mu \mathrm{g}$ every other day from $\mathrm{P} 4$ on. Bars show mean $\pm \mathrm{SD},{ }^{*}=P<0.05,{ }^{*}=P<0.01$. 
Table 1 External phenotype and macroscopic features in P8 SMA mice

\begin{tabular}{|c|c|c|c|c|c|c|c|}
\hline Genotype & Number & Treatment & $\begin{array}{l}\text { Fluid in } \\
\text { abdomen }\end{array}$ & $\begin{array}{l}\text { Lung } \\
\text { findings }\end{array}$ & $\begin{array}{c}\text { Bladder } \\
\text { distension }\end{array}$ & Fur defects & Diarrhea \\
\hline \multirow[t]{11}{*}{ SMA $\left(S m n^{-l-} ; S M N 2\right.$ tg/O $)$} & 1 & None & NA & NA & - & + & +++ \\
\hline & 2 & None & + & NA & - & + & +++ \\
\hline & 6 & None & NA & NA & NA & + & +++ \\
\hline & 7 & None & NA & + & - & + & +++ \\
\hline & 8 & None & $+1-$ & NA & - & + & +++ \\
\hline & 9 & None & + & NA & NA & + & +++ \\
\hline & 10 & None & $+1-$ & NA & - & + & +++ \\
\hline & 5 & JNJ & + & $+1-$ & + & - & + \\
\hline & 6 & JNJ & + & + & + & - & + \\
\hline & 7 & JNJ & + & - & $+1-$ & - & + \\
\hline & 8 & JNJ & - & + & - & - & + \\
\hline \multirow[t]{9}{*}{ Control $\left(S m n^{+/-} ;\right.$SMN2 $\left.{ }^{t g} / 0\right)$} & 3 & None & NA & NA & - & - & - \\
\hline & 4 & None & NA & NA & - & - & - \\
\hline & 5 & None & - & NA & - & - & - \\
\hline & 1 & JNJ & - & - & - & - & - \\
\hline & 2 & JNJ & - & - & - & - & - \\
\hline & 3 & JNJ & - & $+1-$ & - & - & - \\
\hline & 4 & $\mathrm{JNJ}$ & - & - & - & - & - \\
\hline & 10 & JNJ & - & - & - & - & - \\
\hline & 11 & $\mathrm{JNJ}$ & - & - & - & - & - \\
\hline
\end{tabular}

Abbreviations: + , feature observed; $+/-$, possible; - , feature not observed; NA, data not available. Diarrhea:,+++ severe;,++ moderate;,+ mild.

consisted of reduced numbers of villi. Villi in the small intestine were blunt and club-shaped with severe intramural edema in the lamina propria. Epithelial cells showed intracytoplasmic vacuoles predominantly at the tips of the villi and lacteals were occasionally dilated (Figures 4Ad and h). Interestingly, although general outward appearance of the treated animals was improved concerning the lack of fur defects, improved weight gain and activity, and even diarrhea (Table 1), organ impairment was similarly severe at P8 with the exception of intestinal edema, which appeared to be reduced (Supplementary Table S1).

\section{NMJ defects in TVA muscle are ameliorated after JNJ-26481585 treatment in late-stage SMA animals}

The TVA is a muscle known to be severely affected in SMA mouse models and neuromuscular junction disorders. ${ }^{49}$ We examined TVA preparations of SMA and control littermates at P0, P5 and P8 to assess when NMJ defects become apparent. NMJ endplate size of the TVA began to lag behind at P5 in SMA as compared with control animals (Figures 5a, c and d). At P8, we observed striking differences in NMJ endplate size (176 vs $106 \mu \mathrm{m}$; Figures 5a and e). Mean endplate size at P8 in SMA animals was lower than in early symptomatic P5 animals $(130 \mu \mathrm{m})$, suggesting true atrophy rather than size effects due to diminished growth. JNJ-26481585-treated animals do not show any significant differences in endplate size at P5 and endplates are significantly larger in treated than in untreated P8 animals (Figures 5b, d and e).

Skeletal muscle vasculature is severely affected and shows no significant improvement after JNJ-26481585 treatment in earlystage SMA animals

Dramatic deficits in the skeletal muscle capillary bed have been described in another severe $\left(S m n 1^{-1-}\right.$; SMN2) SMA mouse model. ${ }^{46}$ Experiments carried out in an identical manner indicated that a similarly dramatic and significant $(P<0.01)$ decrease $(\sim 29 \%)$ in the skeletal muscle capillary bed of TVA muscle was present at PND5. JNJ-26481585 treatment did not significantly rescue ( $\sim 7 \%$ increase) this vascular defect in the severe Taiwanese SMA mouse model (Supplementary Figure S4).

\section{DISCUSSION}

Elucidation of underlying genes and disease mechanisms has made therapy of genetic diseases a reachable goal in modern drug development. Several HDACi have been shown to upregulate SMN amounts in vitro and in vivo and to ameliorate the SMA phenotype. ${ }^{21-26,29,30,50}$ The novel HDACi JNJ-26481585 has been described to have an improved pharmacodynamic profile with sustained activity. ${ }^{38,39}$ We showed that JNJ-26481585 is able to upregulate SMN protein levels in vitro. Our results in primary patient fibroblast cell lines indicate that, as previously demonstrated for LBH589 and VPA, ${ }^{22,26}$ JNJ-26481585 acts on SMN2 transcription and influences correct splicing. The higher augmentation of SMN protein than SMN2 transcripts is also in line with findings previously reported for VPA and LBH589. ${ }^{26}$ They suggest additional common effects of these HDACi that lead to a stabilization of the SMN protein. Indeed, cancer studies show that the mode of action of HDACi on selected genes is complex and dissociation of RNA and protein levels - eg, by inhibition of proteasomal degradation - has been repeatedly described. ${ }^{51,52}$ In vivo, SMN levels in the spinal cord and brain of late-stage animals was higher than in untreated animals, but levels were still much lower than in the respective control littermates. In QF muscle levels of SMN in both treated and untreated SMA animals were even below detection by western blot analysis. Although onset of motor and weight deficits were delayed in treated animals, the course of disease was not markedly improved and we did not see any extension of life expectancy. Early intervention seems to be of great importance in treatment of SMA and it is likely that a critical SMN level threshold 

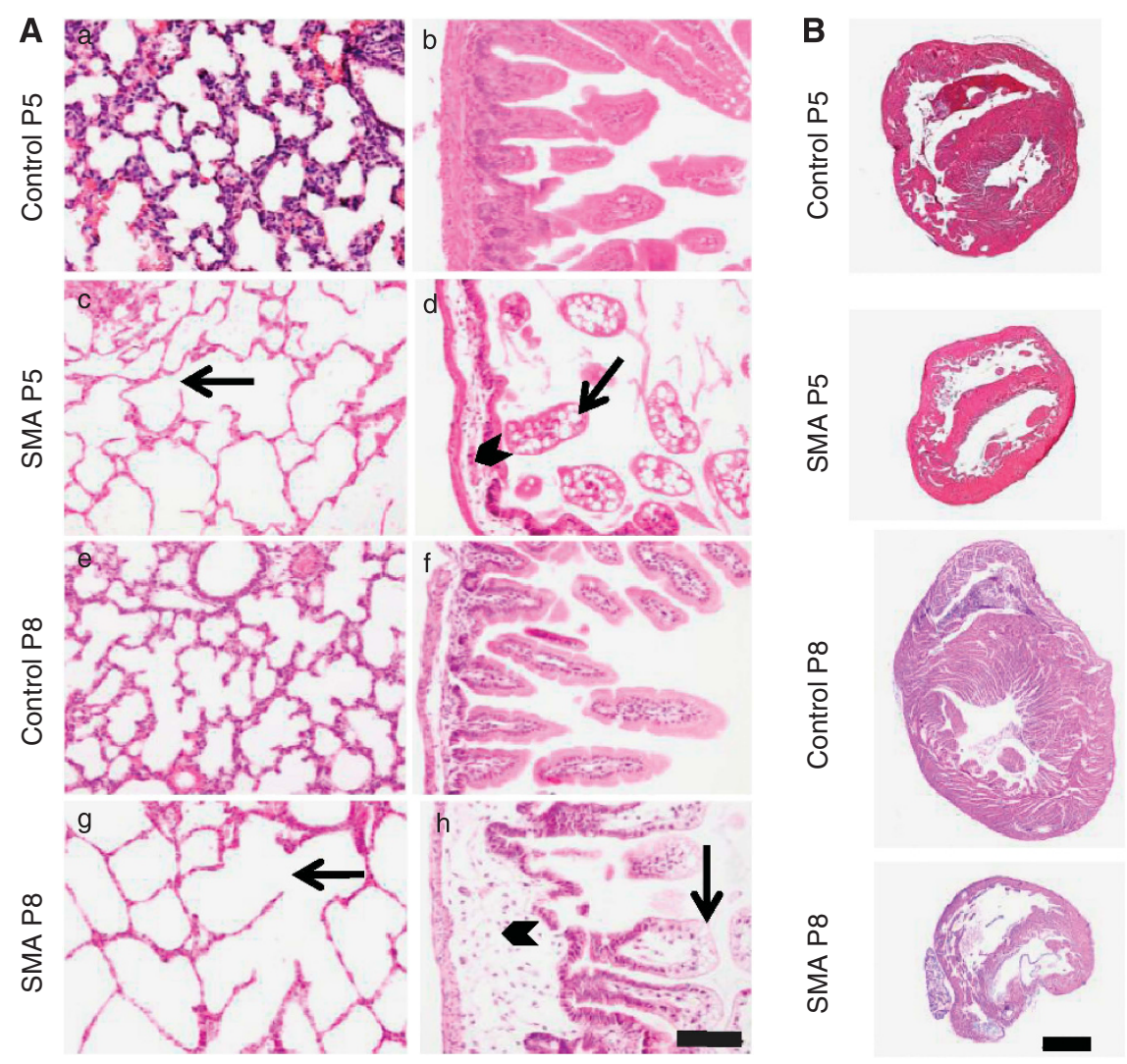

Figure 4 Histology of the lung, intestine and heart of SMA animals compared with control littermates at P5 and P8. (A) Mice at P5 and P8 reveal similar lesions at different degrees of severity. SMA animals (c, g) show variable degrees of emphysema with ruptured alveolar septa and enlarged alveolar spaces compared with control animals $(a, e)$. The small intestine reveals reduced numbers of villi, and villi are blunt and club-shaped with severe intramural edema in the lamina propria of SMA animals (arrowheads in $\mathrm{d}$, h). Mucosal epithelial cells show intracytoplasmatic vacuoles predominantly at the tips of the villi and lacteals were occasionally dilated (arrows in d, h). These lesions are absent in age-matched controls (b, f). Changes were obvious at P5 and show progression towards P8. Scale bar $100 \mu \mathrm{m}$. (B) Representative pictures of hearts of control and SMA animals at P5 and P8. Interventricular septa appear thinned in SMA animals.

exists. ${ }^{18,53-56}$ We aimed to test a situation suitable for future patient care and chose oral application beginning on the first day of life (P0). Protein analysis of SMA animals at different disease stages demonstrates that there may be organ as well as time-dependent effects of HDACi treatment on SMN levels. At early disease stage (P5) there was no SMN upregulation in SMA animals. Possible explanations are that pharmacological properties (eg, half-life) of the drug change during transition from neonatal metabolism to laterstage animals or that there is accumulation of the substance with repeated dosing. On the other hand, the treated animals showed better weight gain and thus the effect of starvation, which was recently described, ${ }^{57}$ to cause an exacerbation of disease also on molecular level by increased exon 7 skipping may be less pronounced in the treated animals. For VPA it has been shown that drug effects are in part due to the induction of additional neuroprotective factors and therefore possibly brought about by different pathways than those directly mediated by increase in SMN. ${ }^{31}$ Finally, non-responsiveness (as also reported for VPA) in some animals is possible. To fully understand the nature and extent of primary and secondary effects of JNJ-26481585 or other HDACi will likely require extensive further investigation. Taken together, protein and phenotypical results suggest that JNJ-26481585 is unable to improve the SMA phenotype to a noteworthy extent in this SMA mouse model under the applied drug regime. We found several gross and histological differences in SMA and control animals that persisted irrespective of treatment. We observed opaque fluid in the abdomen of SMA animals, which was not seen in control animals. Fluid accumulation in the abdominal cavity can occur due to liver failure or stasis of blood in the large ascending veins leading to exudation or transudation and reactive inflammation or due to, eg, intra-abdominal tumors, systemic or local infection. The opaque quality may also indicate lymphatic fluid. Bacteriological and biochemical analysis of the fluid is required to make a definite statement on its origin. Another remarkable gastrointestinal finding in our mice was the often severe diarrhea. Histology of the intestine revealed submucosal edema beginning in early stages of disease. It remains unclear whether accumulation of fluid and the intestinal pathology observed in SMA animals are independent features or are connected. It has been reported that edematous swelling of tissue in other body parts (eg, tail) occurs as part of the phenotype, which was interpreted as a result of progressive failure of the muscle pump to transport venous blood proximally. ${ }^{40}$ Disturbance of the insulin-like growth factor 1 axis has been proposed as explanation for the dwarf-like phenotype of older animals. ${ }^{18,58,59}$ It is possible that the gastrointestinal involvement at least contributes to the diminished growth in SMA animals and impedes effective oral drug treatment in later stages of the disease. Diarrhea or fluid in the abdomen are not commonly described features of SMA in human, but reflux esophagitis is a known feature. ${ }^{60}$ Occasional case 

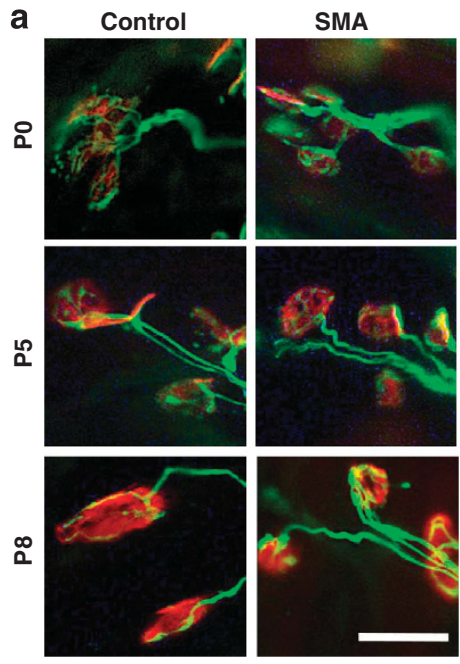

b

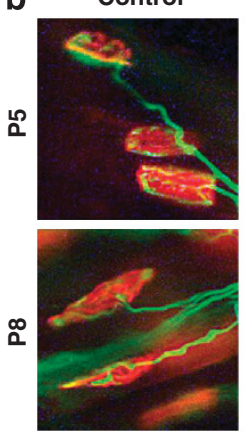

SMA

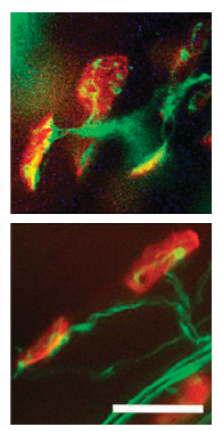

$\infty$

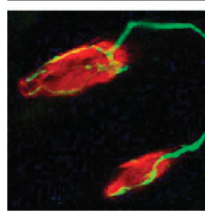

C PO

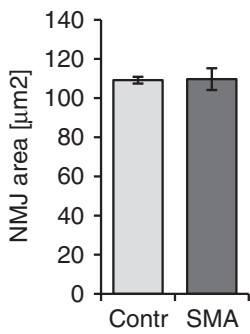

d P5

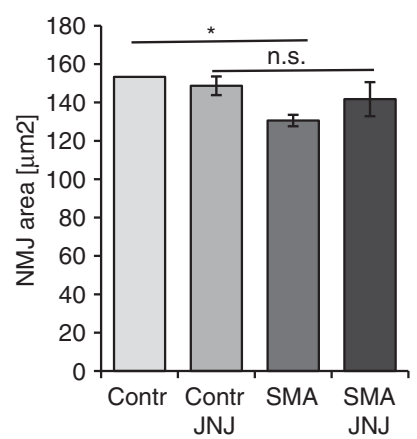

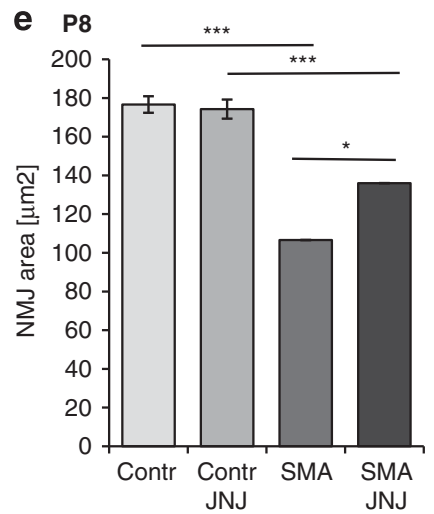

Figure 5 Neuromuscular junctions of SMA and control animals at different disease stages in TVA muscle. (a) Representative pictures of NMJs in untreated SMA mice and control littermates at P0, P5 and P8. Clear size difference between P8 SMA and P8 control animal is shown. Endplates of SMA animals at P8 appear similar in size and shape to PO animals. Synaptic vesicle and neurofilament staining shown in green and bungarotoxin shown in red. (b) Representative pictures of NMJs in JNJ-26481585-treated SMA mice and control littermates at P5 and P8. (c-e) Quantification of the mean area of NMJs at different stages of disease. Growth of NMJs begins to lag behind as early as P5 (d) and NMJ size is even reduced at P8 (e) in affected untreated animals. Treated animals show no significant difference in endplate size at P5 (d) and significantly bigger NMJs at P8 compared with untreated animals (e). Scale bars $25 \mu \mathrm{m}$. Littermates used were as follows: three controls and three SMA at P0, two controls and four SMA at P5, four controls and two SMA at P8. JNJ-26481585-treated animals were as follows: three controls and three SMA at P5, four controls and two SMA at P8. In each animal at least 100 NMJs were quantified. Bars show mean $\pm \mathrm{SD},{ }^{*}=P<0.05,{ }^{* *}=P<0.001$.

reports of constipation later developing into diarrhea as well as diminished haustration of the colon and small intestine distension exist. ${ }^{61,62}$

Acute heart failure explains sudden death. Moreover, heart failure and wasting are known to be bi-directionally related. ${ }^{63}$ The IVS appeared thinned, as did the ventricular walls, in most of our SMA animals at P5 and P8, irrespective of treatment. Owing to methodological limitations (hearts were not uniformly stopped in diastole), we were not able to reliably quantify the degree of morphological heart changes. Cardiomyopathic changes can be rooted or result in rhythm disturbances, which (eg, bradycardia) have been extensively described and seem to be a consistent feature in SMA mice. ${ }^{42,43,64}$ In humans, cardiac involvement has been described in the form of congenital malformations (predominantly SMA type I) and case reports of cardiomyopathy (predominantly SMA type III). ${ }^{41,44,65-67}$ The question remains whether our observations are due to autonomous nerve involvement or muscle development or affection. We found that motoneuron endplate size in the TVA muscle from affected late-stage animals was smaller than that in P0 animals reflecting progressive neurodegeneration. JNJ-26481585-treated animals showed no difference in endplate size at P5 and endplate size was at least retained until P8 corresponding well to the beneficial effect of the HDACi on the motor function of SMA animals. Capillary bed defects were already highly significant in P5 SMA animals and could not be rescued by treatment. As reported before in a different SMA mouse model, ${ }^{46}$ we found that capillary defects did not lag behind NMJ pathology, but rather developed in parallel. It is possible that there is an essential role of SMN deficiency in these tissues and defects are not developing exclusively secondary to $\alpha$-motoneuron deterioration. Further, there is no evidence to suggest that the defects in the autonomic nervous system are likely to affect the patterning of the capillary bed. In fact, sympathetic nerves follow arteries into the periphery ${ }^{68}$ and their development is stunted when endothelial development is blocked. ${ }^{69}$

Massive acidosis offers an explanation for or may contribute to many of the observed pathologies. The lung seems to be affected in early stages of disease in SMA animals. Respiratory failure may result in progressive acidosis leading to multiple organ decompensation 
including the intestinal subcutaneous tissue in the form of edema and stress on the heart. Rapid death may ensue. Future studies should clarify whether this hypothetical cataclysmic chain of events may indeed be responsible for the rapid disease progression observed in our and other SMA models. It will also be interesting to further characterize the complex interplay between SMN depletion, organ pathology and disease progression in different end organs and different disease stages.

In summary, HDACi treatment has repeatedly been shown to be beneficial in mouse models of SMA. Although greater benefit is conceivable for milder affected models of SMA, the effects of the tested HDACi JNJ-26481585 cannot overcome the pathology observed in our severely affected animals. The phenotypic peculiarities found in this study, together with other emerging reports, give a strong hint to possible primary or secondary involvement of organs previously thought to be largely unaffected at least early in this disease. The findings described here need corroboration and further characterization also in other mouse models of SMA to establish whether they represent an inherent part of the phenotype. Better understanding of organ involvement may eventually lead to improved standard of care by closer monitoring of SMA patients regarding development of respective pathology and early intervention.

\section{CONFLICT OF INTEREST}

The authors declare no conflict of interest.

\section{ACKNOWLEDGEMENTS}

This work was supported by grants provided from the Deutsche Forschungsgemeinschaft to BW (Wi-945/12-3).

1 Crawford TO, Pardo CA: The neurobiology of childhood spinal muscular atrophy. Neurobiol Dis 1996; 3: 97-110.

2 Feldkotter M, Schwarzer V, Wirth R, Wienker TF, Wirth B: Quantitative analyses of SMN1 and SMN2 based on real-time lightCycler PCR: fast and highly reliable carrier testing and prediction of severity of spinal muscular atrophy. Am J Hum Genet 2002; 70: $358-368$.

3 Thieme A, Mitulla B, Schulze F, Spiegler AW: Chronic childhood spinal muscular atrophy in Germany (West-Thuringen)-an epidemiological study. Hum Genet 1994; 93: 344-346.

4 Lefebvre S, Burglen L, Reboullet S et al: Identification and characterization of a spinal muscular atrophy-determining gene. Cell 1995; 80: 155-165.

5 Wirth B: An update of the mutation spectrum of the survival motor neuron gene (SMN1) in autosomal recessive spinal muscular atrophy (SMA). Hum Mutat 2000; 15 228-237.

6 Alias L, Bernal S, Fuentes-Prior P et al: Mutation update of spinal muscular atrophy in Spain: molecular characterization of 745 unrelated patients and identification of four novel mutations in the SMN1 gene. Hum Genet 2009; 125: 29-39.

7 Munsat TL, Davies KE: International SMA consortium meeting. (26-28 June 1992, Bonn, Germany). Neuromuscul Disord 1992; 2: 423-428.

8 McAndrew PE, Parsons DW, Simard LR et al: Identification of proximal spinal muscular atrophy carriers and patients by analysis of SMNT and SMNC gene copy number. Am J Hum Genet 1997; 60: 1411-1422.

9 Wirth B, Brichta L, Schrank B et al: Mildly affected patients with spinal muscular atrophy are partially protected by an increased SMN2 copy number. Hum Genet 2006; 119: 422-428.

10 Kashima T, Manley JL: A negative element in SMN2 exon 7 inhibits splicing in spinal muscular atrophy. Nat Genet 2003; 34: 460-463.

11 Lorson CL, Hahnen E, Androphy EJ, Wirth B: A single nucleotide in the SMN gene regulates splicing and is responsible for spinal muscular atrophy. Proc Natl Acad Sci USA 1999; 96: 6307-6311.

12 Cartegni L, Krainer AR: Disruption of an SF2/ASF-dependent exonic splicing enhance in SMN2 causes spinal muscular atrophy in the absence of SMN1. Nat Genet 2002; 30: $377-384$

13 Helmken C, Hofmann Y, Schoenen $\mathrm{F}$ et al: Evidence for a modifying pathway in SMA discordant families: reduced SMN level decreases the amount of its interacting partners and Htra2-beta1. Hum Genet 2003; 114: 11-21.

14 Lorson CL, Androphy EJ: An exonic enhancer is required for inclusion of an essential exon in the SMA-determining gene SMN. Hum Mol Genet 2000; 9: 259-265.

15 Wirth B, Brichta L, Hahnen E: Spinal muscular atrophy and therapeutic prospects. Prog Mol Subcell Biol 2006; 44: 109-132.
16 Lorson CL, Rindt H, Shababi M: Spinal muscular atrophy: mechanisms and therapeutic strategies. Hum Mol Genet 2010; 19: R111-R118.

17 Burnett BG, Crawford TO, Sumner CJ: Emerging treatment options for spinal muscular atrophy. Curr Treat Options Neurol 2009; 11: 90-101.

18 Hua Y, Sahashi K, Rigo F et al: Peripheral SMN restoration is essential for long-term rescue of a severe spinal muscular atrophy mouse model. Nature 2011; 478: $123-126$

19 Wadman RI, Bosboom WM, van den Berg LH, Wokke JH, lannaccone ST, Vrancken AF: Drug treatment for spinal muscular atrophy types II and III. Cochrane Database Syst Rev 2011: 12: CD006282.

20 Wadman RI, Bosboom WM, van den Berg LH, Wokke JH, lannaccone ST, Vrancken AF: Drug treatment for spinal muscular atrophy type I. Cochrane Database Syst Rev 2011; 12: CD006281.

21 Brichta L, Holker I, Haug K, Klockgether T, Wirth B: In vivo activation of SMN in spinal muscular atrophy carriers and patients treated with valproate. Ann Neurol 2006; 59: 970-975.

22 Brichta L, Hofmann Y, Hahnen E et al: Valproic acid increases the SMN2 protein level: a well-known drug as a potential therapy for spinal muscular atrophy. Hum Mol Genet 2003; 12: 2481-2489.

23 Sumner CJ, Huynh TN, Markowitz JA et al: Valproic acid increases SMN levels in spinal muscular atrophy patient cells. Ann Neurol 2003; 54: 647-654.

24 Hahnen E, Eyupoglu IY, Brichta L et al: In vitro and ex vivo evaluation of secondgeneration histone deacetylase inhibitors for the treatment of spinal muscular atrophy. J Neurochem 2006; 98: 193-202.

25 Riessland M, Brichta L, Hahnen E, Wirth B: The benzamide M344, a novel histone deacetylase inhibitor, significantly increases SMN2 RNA/protein levels in spinal muscular atrophy cells. Hum Genet 2006; 120: 101-110.

26 Garbes L, Riessland M, Holker I et al: LBH589 induces up to 10-fold SMN protein levels by several independent mechanisms and is effective even in cells from SMA patients non-responsive to valproate. Hum Mol Genet 2009; 18: 3645-3658.

27 Chang JG, Hsieh-Li HM, Jong YJ, Wang NM, Tsai CH, Li H: Treatment of spinal muscular atrophy by sodium butyrate. Proc Natl Acad Sci USA 2001; 98: 9808-9813.

28 Kernochan LE, Russo ML, Woodling NS et al: The role of histone acetylation in SMN gene expression. Hum Mol Genet 2005; 14: 1171-1182.

29 Avila AM, Burnett BG, Taye AA et al: Trichostatin A increases SMN expression and survival in a mouse model of spinal muscular atrophy. J Clin Invest 2007; 117: 659-671.

30 Riessland M, Ackermann B, Forster A et al: SAHA ameliorates the SMA phenotype in two mouse models for spinal muscular atrophy. Hum Mol Genet 2010; 19 : 2154-2167

31 Tsai LK, Tsai MS, Ting CH, Li H: Multiple therapeutic effects of valproic acid in spinal muscular atrophy model mice. J Mol Med 2008; 86: 1243-1254.

32 Piepers S, Cobben JM, Sodaar P et al: Quantification of SMN protein in leucocytes from spinal muscular atrophy patients: effects of treatment with valproic acid. $J$ Neurol Neurosurg Psychiatry 2011; 82: 850-852.

33 Weihl CC, Connolly AM, Pestronk A: Valproate may improve strength and function in patients with type III/IV spinal muscle atrophy. Neurology 2006; 67: 500-501.

34 Kissel JT, Scott CB, Reyna SP et al: SMA CARNIVAL TRIAL PART II: a prospective, single-armed trial of L-carnitine and valproic acid in ambulatory children with spinal muscular atrophy. PLoS One, 2011; 6: e21296.

35 Swoboda KJ, Scott CB, Crawford TO et al: SMA CARNI-VAL trial part I: double-blind, randomized, placebo-controlled trial of L-carnitine and valproic acid in spinal muscular atrophy. PLoS One 2011; 5: e12140.

36 Tong WG, Wei $Y$, Stevenson $W$ et al: Preclinical antileukemia activity of JNJ26481585, a potent second-generation histone deacetylase inhibitor. Leuk Res 2010; 34: 221-228.

37 Deleu S, Lemaire M, Arts J et al: The effects of JNJ-26481585, a novel hydroxamatebased histone deacetylase inhibitor, on the development of multiple myeloma in the 5T2MM and 5T33MM murine models. Leukemia 2009; 23: 1894-1903.

38 Arts J, King P, Marien A et al: JNJ-26481585, a novel 'second-generation' oral histone deacetylase inhibitor, shows broad-spectrum preclinical antitumoral activity. Clin Cancer Res 2009; 15: 6841-6851.

39 Stuhmer T, Arts J, Chatterjee $\mathrm{M}$ et al: Preclinical anti-myeloma activity of the novel HDAC-inhibitor JNJ-26481585. Br J Haematol 2010; 149: 529-536.

40 Hsieh-Li HM, Chang JG, Jong YJ et al: A mouse model for spinal muscular atrophy. Nat Genet 2000; 24: 66-70.

41 Menke LA, Poll-The BT, Clur SA et al: Congenital heart defects in spinal muscular atrophy type I: a clinical report of two siblings and a review of the literature. Am J Med Genet A 2008; 146A: 740-744.

42 Heier CR, Satta R, Lutz C, DiDonato CJ: Arrhythmia and cardiac defects are feature of spinal muscular atrophy model mice. Hum Mol Genet 2010; 19 : 3906-3918.

43 Shababi M, Habibi J, Yang HT, Vale SM, Sewell WA, Lorson CL: Cardiac defects contribute to the pathology of spinal muscular atrophy models. Hum Mol Genet 2010; 19: 4059-4071.

44 Rudnik-Schoneborn S, Heller R, Berg C et al: Congenital heart disease is a feature of severe infantile spinal muscular atrophy. J Med Genet 2008; 45: 635-638.

45 Mutsaers CA, Wishart TM, Lamont DJ et al: Reversible molecular pathology of skeletal muscle in spinal muscular atrophy. Hum Mol Genet 2011; 20: 4334-4344.

46 Somers E, Stencel Z, Wishart TM, Gillingwater TH, Parson SH: Density, calibre and ramification of muscle capillaries are altered in a mouse model of severe spinal muscular atrophy. Neuromuscul Disord 2012; 22: 435-442. 
47 Mende $\mathrm{Y}$, Jakubik M, Riessland $\mathrm{M}$ et al: Deficiency of the splicing factor Sfrs 10 results in early embryonic lethality in mice and has no impact on full-length SMN/Smn splicing. Hum Mol Genet 2010; 19: 2154-2167.

48 El-Khodor BF, Edgar N, Chen A et al: Identification of a battery of tests for drug candidate evaluation in the SMNDelta7 neonate model of spinal muscular atrophy. Exp Neurol 2008; 212: 29-43.

49 Murray LM, Comley LH, Thomson D, Parkinson N, Talbot K, Gillingwater TH: Selective vulnerability of motor neurons and dissociation of pre- and post-synaptic pathology at the neuromuscular junction in mouse models of spinal muscular atrophy. Hum $\mathrm{Mol}$ Genet 2008; 17: 949-962.

50 Hauke J, Riessland M, Lunke S et al: Survival motor neuron gene 2 silencing by DNA methylation correlates with spinal muscular atrophy disease severity and can be bypassed by histone deacetylase inhibition. Hum Mol Genet 2009; 18 304-317.

51 Borbone E, Berlingieri MT, De Bellis F et al: Histone deacetylase inhibitors induce thyroid cancer-specific apoptosis through proteasome-dependent inhibition of TRAIL degradation. Oncogene 2012; 29: 105-116.

52 Yeung A, Bhargava RK, Ahn R et al: HDAC inhibitor M344 suppresses MCF-7 breast cancer cell proliferation. Biomed Pharmacother 2010; 66: 232-236.

53 Passini MA, Bu J, Richards AM et al: Antisense oligonucleotides delivered to the mouse CNS ameliorate symptoms of severe spinal muscular atrophy. Sci Trans/ Med 2011; 3: 72ra18.

54 Bowerman M, Murray LM, Beauvais A, Pinheiro B, Kothary R: A critical Smn threshold in mice dictates onset of an intermediate spinal muscular atrophy phenotype associated with a distinct neuromuscular junction pathology. Neuromuscul Disord 2012; 22: 263-276.

55 Murray LM, Lee S, Baumer D, Parson SH, Talbot K, Gillingwater TH: Pre-symptomatic development of lower motor neuron connectivity in a mouse model of severe spinal muscular atrophy. Hum Mol Genet 2010; 19: 420-433.

56 Porensky PN, Mitrpant C, McGovern VL et al: A single administration of morpholino antisense oligomer rescues spinal muscular atrophy in mouse. Hum Mol Genet 2012; 21: $1625-1638$
57 Sahashi K, Hua Y, Ling KK et al: TSUNAMI: an antisense method to phenocopy splicing-associated diseases in animals. Genes Dev 2012; 26: 1874-1884.

58 Kunkel SD, Suneja M, Ebert SM et al: mRNA expression signatures of human skeletal muscle atrophy identify a natural compound that increases muscle mass. Cell Metab 2011; 13: 627-638.

59 Bosch-Marce M, Wee CD, Martinez TL et al: Increased IGF-1 in muscle modulates the phenotype of severe SMA mice. Hum Mol Genet 2011; 20: 1844-1853.

60 Yuan N, Wang CH, Trela A, Albanese CT: Laparoscopic Nissen fundoplication during gastrostomy tube placement and noninvasive ventilation may improve survival in type and severe type II spinal muscular atrophy. J Child Neurol 2007; 22: 727-731.

61 Karasick D, Karasick S, Mapp E: Gastrointestinal radiologic manifestations of proximal spinal muscular atrophy (Kugelberg-Welander syndrome). J Natl Med Assoc 1982; 74: 475-478.

62 Ionasescu V, Christensen J, Hart M: Intestinal pseudo-obstruction in adult spinal muscular atrophy. Muscle Nerve 1994; 17: 946-948.

63 Lainscak M, Podbregar M, Anker SD: How does cachexia influence survival in cancer, heart failure and other chronic diseases? Curr Opin Support Palliat Care 2007; 1: 299-305.

64 Bevan AK, Hutchinson KR, Foust KD et al: Early heart failure in the SMNDelta7 mode of spinal muscular atrophy and correction by postnatal scAAV9-SMN delivery. Hum Mol Genet 2010; 19: 3895-3905.

65 Roos M, Sarkozy A, Chierchia GB, De Wilde P, Schmedding E, Brugada P: Malignant ventricular arrhythmia in a case of adult onset of spinal muscular atrophy (KugelbergWelander disease). J Cardiovasc Electrophysiol 2009; 20: 342-344.

66 Takahashi N, Shimada T, Ishibashi Y et al: Cardiac involvement in Kugelberg-Welander disease: a case report and review. Am J Med Sci 2006; 332: 354-356.

67 Vaidla E, Talvik I, Kulla A et al: Neonatal spinal muscular atrophy type 1 with bone fractures and heart defect. J Child Neurol 2007; 22: 67-70.

68 Glebova NO, Ginty DD: Growth and survival signals controlling sympathetic nervous system development. Annu Rev Neurosci 2005; 28: 191-222.

69 Honma Y, Araki T, Gianino $\mathrm{S}$ et al: Artemin is a vascular-derived neurotropic factor for developing sympathetic neurons. Neuron 2002; 35: 267-282.

Supplementary Information accompanies the paper on European Journal of Human Genetics website (http://www.nature.com/ejhg) 